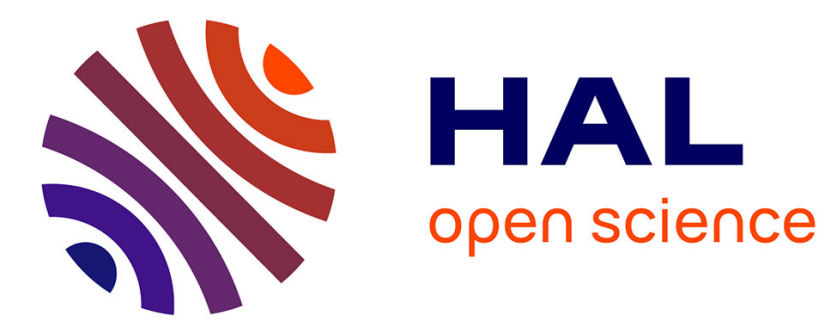

\title{
Optimum Design of LPCVD Reactors
}

L. Zambov

\section{To cite this version:}

L. Zambov. Optimum Design of LPCVD Reactors. Journal de Physique IV Proceedings, 1995, 05 (C5), pp.C5-269-C5-276. 10.1051/jphyscol:1995531 . jpa-00253857

\section{HAL Id: jpa-00253857 https://hal.science/jpa-00253857}

Submitted on 1 Jan 1995

HAL is a multi-disciplinary open access archive for the deposit and dissemination of scientific research documents, whether they are published or not. The documents may come from teaching and research institutions in France or abroad, or from public or private research centers.
L'archive ouverte pluridisciplinaire HAL, est destinée au dépôt et à la diffusion de documents scientifiques de niveau recherche, publiés ou non, émanant des établissements d'enseignement et de recherche français ou étrangers, des laboratoires publics ou privés. 


\title{
Optimum Design of LPCVD Reactors
}

\author{
L.M. Zambov
}

Sofia University of Technology, Department of Semiconductors, 8 Kliment Ohridski Blvd., 1756 Sofia, Bulgaria

\begin{abstract}
A general mathematical model for the description of heat transfer and mass transport processes in a hotwall multiple-wafer horizontal tube LPCVD reactor is developed. Investigations on the influence of the geometric characteristics of the reactor system on the thickness uniformity of the layers and on the economically expedient performance of the processes are carried out by the model. In this way, information about the optimum geometry of the reactor is obtained. Technical modifications of the conventional LPCVD systems are analyzed which allow the obtainment of uniform layers under conditions of mass production without applying temperature difference along the reaction zone. A model of injection-fed reactors is built that helps make conclusions about the optimum design of the injectors when they are used in a regime of maintaining constant concentration in the system.
\end{abstract}

\section{INTRODUCTION}

The efforts for achieving high technical and technological parameters in Chemical Vapour Deposition (CVD) have led to wide implementation of the methods of mathematical modeling. In the majority of investigations, devoted to CVD modeling, the ultimate purpose is the analysis of the model in view of estimating the influence of various technological parameters on particular chemical processes [1-4]. A number of works $[5,6]$ are related to the optimization of the processes as a final aim of the modeling. At the same time, the design of CVD reactors is an object of a scarce number of investigations $[7,8]$. According to different authors $[9,10]$, it is accomplished empirically, processing and analyzing a vast quantity of experimental results. Therefore, it is interesting to present and study analytically the universal relationships between the characteristics of the reactor design and the process parameters.

The large scale of integration of modern microelectronic devices is achieved, on one hand, through expansion of the chip area, and on the other hand, through reduction of the sizes of the elements and the distances between them. The increase in throughput, in turn, requires the use of monocrystalline wafers with larger diameters. For these reasons, under conditions of mass production, the requirements for uniformity of thickness and composition of the deposited layers becomes a crucial factor, governing to a large extent the quality of the microelectronic devices. That is why, it is mandatory that CVD systems be designed in which the problem of layer non-uniformity is effectively and cheaply solved. The solution of the problems posed determines the aim of the present investigation.

\section{RESULTS AND DISCUSSION}

\subsection{Design of conventional LPCVD reactors}

The basic geometric characteristics of the system are length $(L)$ and radius $\left(R_{\mathrm{T}}\right)$ of the quartz tube of the reactor (Fig. 1), radius of the vertically positioned monocrystalline wafers $\left(R_{w}\right)$ and distance $(H)$ between them. The length of the quartz tube $(L)$ is dictated by the temperature of the processes taking place and by the length of the temperature plateau of the reactor $\left(L_{3}\right)$ (Fig. 2). The latter depends on the number of wafers $(n)$ and the distance $(H)$ between them. In terms of the temperature field, several temperature regions can be distinguished in a LPCVD reactor [11]. In industrial systems, the length $L_{1}$ of the first zone 


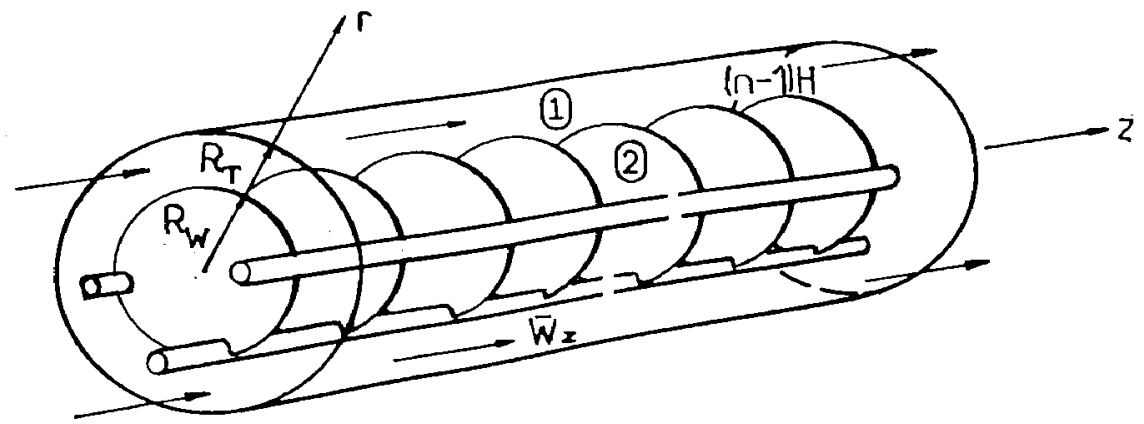

Fig. 1. Schematic of conventional LPCVD reactor.

(on both sides of the temperature plateau) is technically limited and is determined by the temperature stability of the vacuum sealings of the system. The distance $L_{2}$ (Fig.2) defines the interval during which the gas flow has reached the temperature of the reactor plateau and the flow has become steady. The exact determination of $L_{2}$.demands solving the differential equation of connective heat transfer [11]. Numerical experiments show that the length of the second temperature zone varies in the range $8-14 \mathrm{~cm}$.

Useful information about the optimum geometry of the reactor in view of satisfying the requirements for uniform layer thickness can be obtained, if a simplified diffusion-kinetic mechanism is assumed on whose basis the differential equations for mass transport are solved:

- for the region between the individual wafers:

$\frac{1}{r} \frac{d}{d r}\left(r \frac{d C}{d r}\right)=\frac{2 k C}{D H}$

with boundary conditions:

$$
\begin{aligned}
& r=0 ; \frac{d C}{d r}=0 \\
& r=R_{\mathrm{w}} ; C=C_{\mathrm{r}=\mathrm{R}_{\mathrm{w}}} ;
\end{aligned}
$$

- for the region around the wafers

$$
\left[D d\left(\frac{d C}{d z}\right)-\bar{w}_{z} d C\right]\left(R_{\mathrm{T}}^{2}-R_{\mathrm{w}}^{2}\right)=\left(k C 2 R_{\mathrm{T}}+D \frac{\partial C}{\left.\partial\right|_{\mathrm{r}=\mathrm{R}_{\mathrm{w}}}} \cdot 2 R_{\mathrm{w}}\right) d z
$$

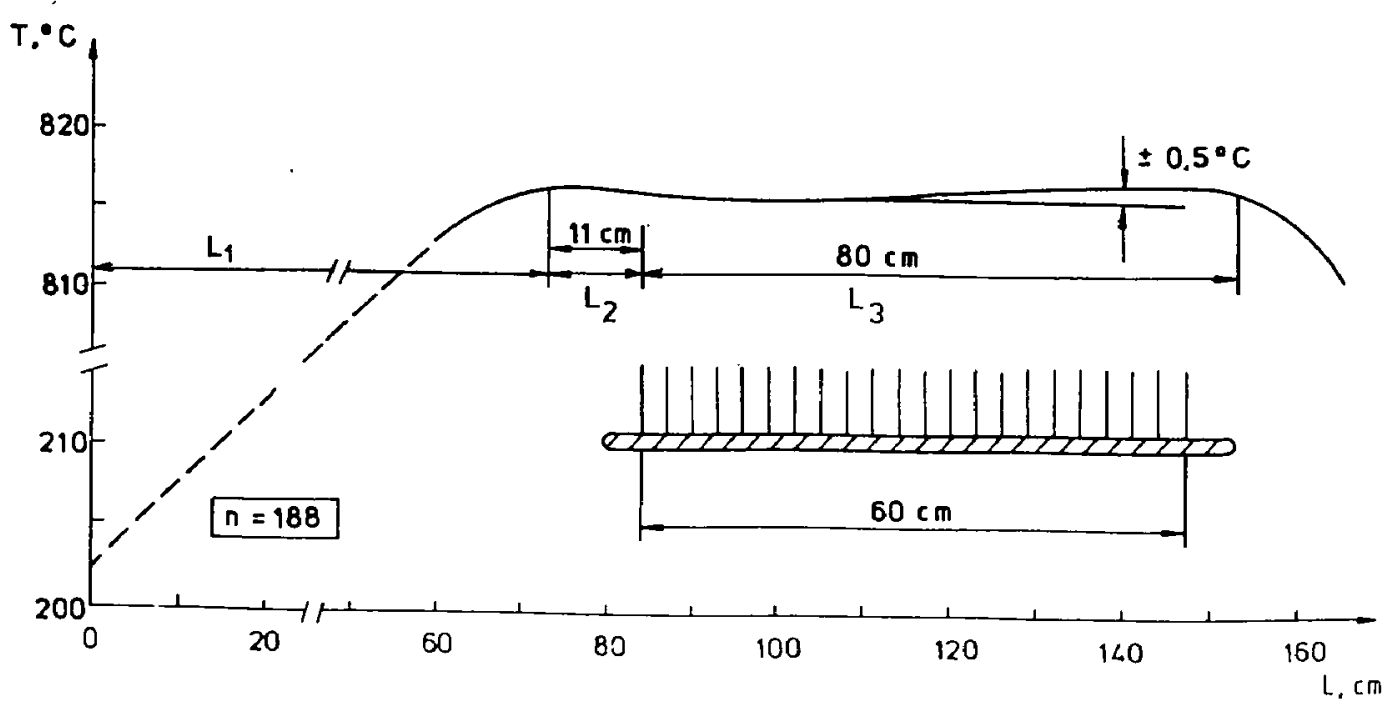

Fig. 2. Temperature profile of the oven and details of wafer positioning for a process $\mathrm{Si}_{3} \mathrm{~N}_{4}$ growth (T=815 $\pm 0.5{ }^{\circ} \mathrm{C}$. 
with boundary conditions:

$$
\begin{aligned}
& z=0 ; C=C^{0} \\
& z \rightarrow \infty ; C \rightarrow 0
\end{aligned}
$$

The analytical solution of eq. (1-4) has the following form:

$$
C(r, z)=C^{0} \frac{I_{0}\left(u \frac{r}{R_{\mathrm{w}}}\right)}{I_{0}(u)} \cdot \exp \left[\left(\frac{\mathrm{Pe}}{2}-\sqrt{\left(\frac{\mathrm{Pe}}{2}\right)^{2}+G\left(\operatorname{Sh} \frac{R_{\mathrm{T}}}{H}+u \frac{I_{1}(u)}{I_{0}(u)}\right)}\right)(j-1)\right]
$$

where $u=\sqrt{2 \mathrm{Sh}} \cdot \frac{R_{\mathrm{w}}}{H} ; G=2 H^{2} /\left(R_{\mathrm{T}}^{2}-R_{\mathrm{w}}^{2}\right) ; \mathrm{Sh}=\frac{k H}{D} ; \mathrm{Pe}=\frac{\bar{w}_{\mathrm{z}} H}{D}$.

It follows from (5) that the radial non-uniformity $\delta_{\mathrm{r}}$ depends on Sherwood's criterion (Sh), $R_{\mathrm{w}}$ and $H$, and the axial non-uniformity $\delta_{z}$ - on Sh, Pecklet's criterion $(\mathrm{Pe})$ and all geometric characteristics of the system. Fig. 3 illustrates the influence of the dimensionless distance $H / R_{\mathrm{w}}$ on the radial non-uniformity of the layers thickness for various values of Sh. Invariance in the relationships about $\delta_{\mathrm{r}}$ is observed at values of $H / R_{\mathrm{w}}$ within $0.04-0.1$. It should be pointed out that in industrial reactors for layer deposition, the ratio $H / R_{w}$ is maintained in these limits. From Fig. 3 it is also evident that a process at $\mathrm{Sh}>10^{-3}$ is inexpedient, since the condition $\delta_{\mathrm{r}}<5 \%$, enforced by practice, is not satisfied.

The influence of the criteria of Sherwood and Pecklet is also illustrated in Fig.4, where the axial nonuniformity $\delta_{\mathrm{z}}$ is plotted as a function of the reactor radius $R_{\mathrm{T}}$. It is seen that after a certain value, the increase in $R_{\mathrm{T}}$ does not lead to a substantial improvement of the coating uniformity, but causes excessive consumption of the reagent. This radius is about $10 \mathrm{~cm}$ and coincides with the internal radius of industrial reactors for layer deposition on wafers with a diameter of $10-12 \mathrm{~cm}$.

It can be summarized that the analysis of the model reveals a fair agreement between the predicted and the practical geometric characteristics of the reactors.

\subsection{Design of LPCVD reactors with reversible flow}

The problem of axial non-uniformity $\delta_{z}$ in conventional reactors is solved by setting a temperature profile along the reaction zone [4]. However, the investigations reveal [12-14] that the properties and the structure of the layers are often strongly temperature-dependent and differences in temperature lead to substantial deviations in the parameters of the produced coatings. This is unacceptable for conditions of series treatment of the wafers.

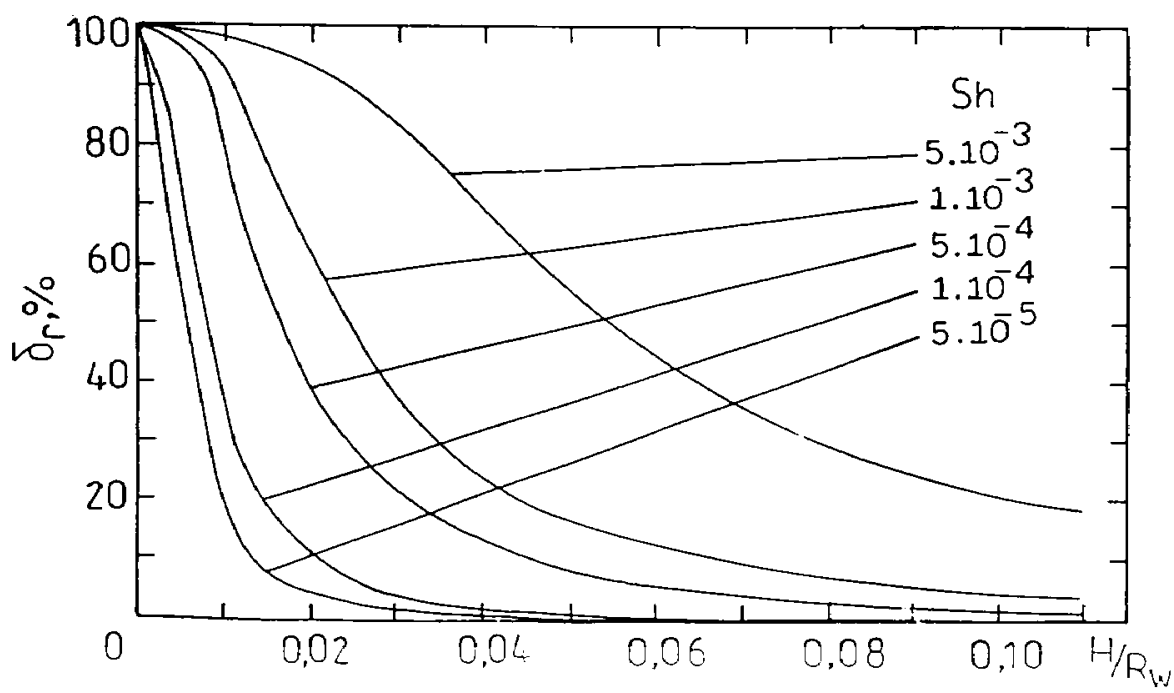

Fig. 3. Dependence of the radial non-uniformity $(\delta$,$) on the geometric factor H / R_{x}$ for different values of Shenvood's criterion (Sh) 


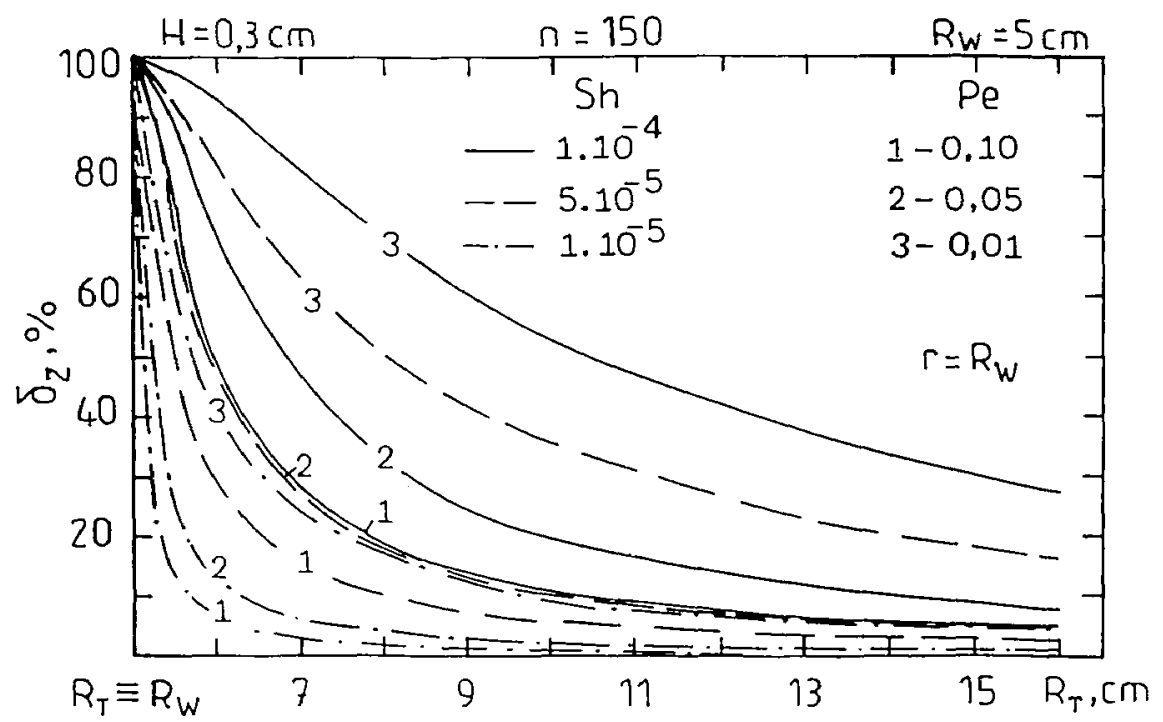

Fig.4. Dependence of the axial non-uniformity $\delta_{z}$ on the internal radius of the quartz tube of the reactor ( $R_{T}$ ) for different values of $\mathrm{Sh}$ and $\mathrm{Pe}$.

Fig. 5 displays a layout of a LPCVD reactor that is free of this shortcoming. The gas distribution unit of the system is provided with piping that ensure two-way feeding and pumping of the reagents.

The problem of axial non-uniformity of the layers in reactors of this type can be dealt with through successive change in the direction of feeding and pumping of the gas flow. This could result in periodic reversible change of the concentration gradient in the reaction zone, and hence, in the deposition of coatings with uniform thickness.

Fig.6a,b illustrates the results from the simulation of two deposition processes using the described method

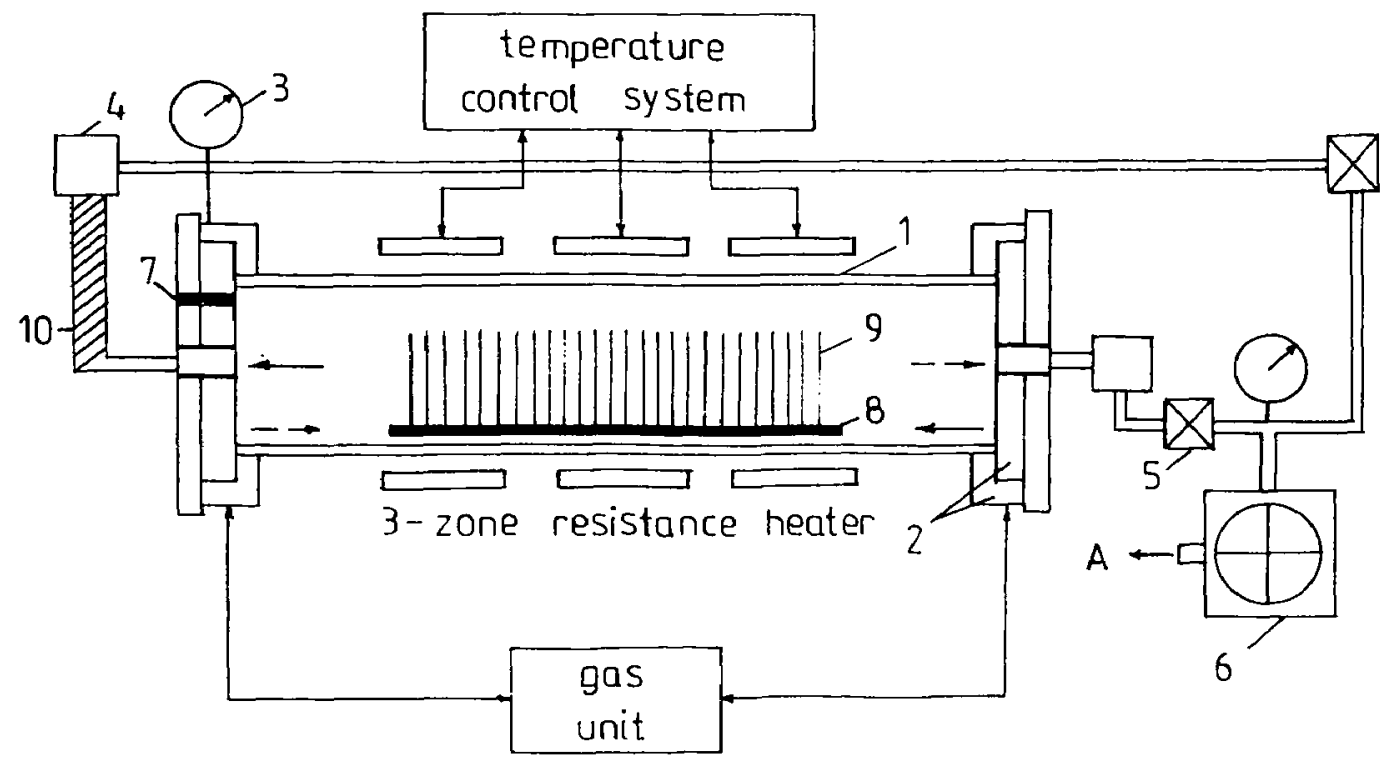

Fig. 5. Configuration of LPCVD reactor system with reversible feeding. 


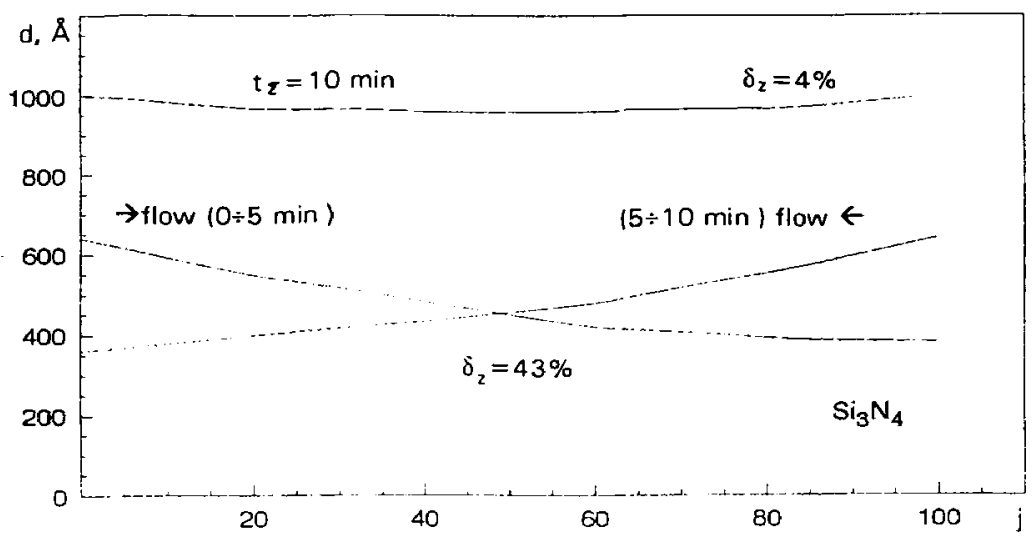

Fig. 6a. Simulation data for the thickness (d) of $\mathrm{Si}_{3} N_{4}$ films grown at the conditions of reversible flow as a function of axial position of wafers $(\mathrm{j}): R_{T}=5.75 \mathrm{~cm}, R_{w}=3.81 \mathrm{~cm}, X_{D C S}^{0}=0.154, \mathrm{P}=75 \mathrm{~Pa}, V_{\Sigma}^{0}=182 \mathrm{sccm}, \mathrm{T}=840^{\circ} \mathrm{C}$.

- polysilicon and silicon nitride with thicknesses $4000 \AA$ and $1000 \AA$, respectively. It is seen from the figure that the predicted results for the thicknesses have $\delta_{z}$ up to $4 \%$.

It should be pointed out that the reactor system in Fig. 5 is easy to be realized technically.

\subsection{Design of LPCVD reactors with injection systems}

The reactors with reversible feeding allow alteration of the concentration gradient but do not eliminate it altogether. The presence of a concentration difference leads to structural changes [14], and to changes in the layer composition when multi-component gas mixtures are used (for deposition of doped polysilicon, silicate glasses, etc.). The up-to-date solution of this problem is the utilization of reactors with injection feeding

The mathematical description of the injection reactors is simplified considerably by the fact that the axial mixing can be disregarded. Then, in accordance with the law of mass conservation

$$
d G_{\mathrm{c}}+d G_{\mathrm{a}}=d G,
$$

where $d G$ is the sum of the local and convective change of the quantity of the limiting component in an elementary volume, $d G_{\mathrm{c}}$ is the quantity of the active component, consumed by an internal volume consumer (the chemical reaction) in an elementary volume, and $\mathrm{dG}_{\mathrm{a}}$ is the augmentation in the quantity of the active component resulting from injection in the elementary volume.

In the presence of main gas flow in the system, as is the general case, it follows from (6) that

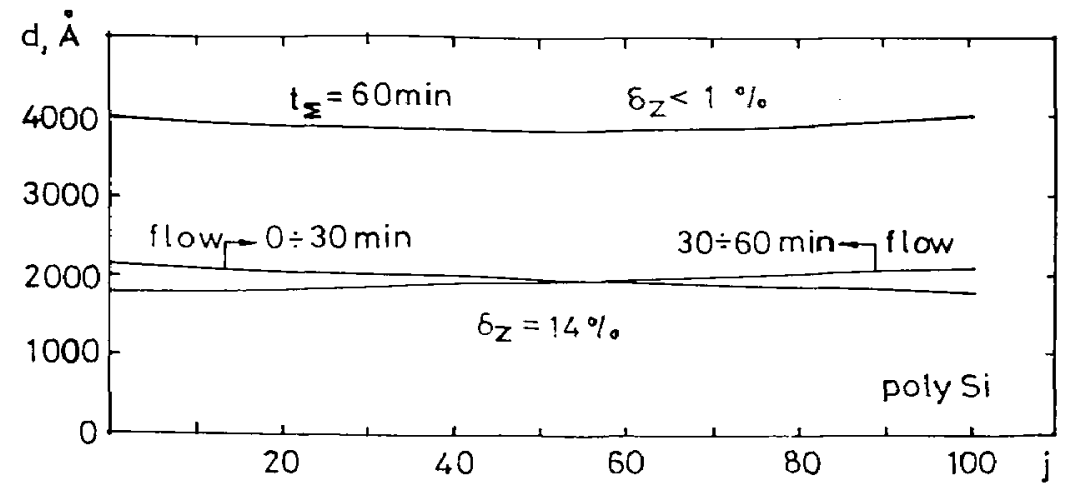

Tig. 6b. Thickness (d) of polysilicon films as a function of wafer position ( $i$ ) for a process simulated under the conditions of reversible fecding: $R_{7}=9 \mathrm{~cm}, R_{\mathrm{w}}=5 \mathrm{~cm}, \mathrm{P}=53 \mathrm{~Pa}, V=150 \operatorname{sccm} . \mathrm{T}=600{ }^{\circ} \mathrm{C} . \mathrm{H}=0.4 \mathrm{~cm}$ 
$-\bar{w}_{\mathrm{z}} d C \cdot \pi\left(R_{\mathrm{T}}^{2}-R_{\mathrm{w}}^{2}\right)=2 J\left(z, R_{\mathrm{w}}\right) \cdot \pi\left(R_{\mathrm{T}}+\frac{R_{\mathrm{w}}^{2}}{H}\right) d z-\frac{V_{\mathrm{g}} \cdot \rho_{\mathrm{g}}}{M_{\mathrm{g}}} \frac{d \mathrm{z}}{\mathrm{H}}$

The numerical solution of (7) for the process of polysilicon deposition, taking into account the dilution, is the following set of algebraic equations:

$$
\begin{aligned}
& \eta(0)=0 ; \\
& \eta(j-1)=\frac{\sum_{\mathrm{k}=1}^{\mathrm{j}-1}\left[R_{\mathrm{D}}(k) S(k)\right] \cdot \rho \cdot 22,4}{M V_{\Sigma}(j-1) x^{0}} ; \\
& R_{\mathrm{D}}(j)=\frac{M}{\rho} k_{\mathrm{p}} k_{\mathrm{SiH}_{4}} \frac{P}{R T} \frac{[1-\eta(j-1)+\beta(j)]}{[1-\alpha \eta(j-1)]},\left[1+k_{\mathrm{SiH}_{4}} \frac{P}{R T} \frac{[1-\eta(j-1)+\beta(j)]}{[1-\alpha \eta(j-1)]}\right]
\end{aligned}
$$

where

$$
x_{\mathbf{g}}^{\mathrm{i}}=\sum_{\mathbf{k}=1}^{\mathrm{j}} \frac{V_{\mathbf{g}}^{\mathrm{i}}(k)}{V_{\Sigma}(k)} ; \beta(j)=\frac{x_{\mathrm{g}}^{\mathrm{i}}}{x_{0}} ; \quad V_{\Sigma}(j)=V_{\Sigma}^{\mathrm{o}}\left[x^{\mathrm{o}}(1-\alpha \eta(j-1)+\beta(j))\right]
$$

Fig.7 shows the results from the model about the deposition rate at three different regimes with (1) and without (2) injection feeding of the active component $\left(100 \% \mathrm{SiH}_{4}\right)$. The same figure shows the axial change in the gas flow of the injected silane, necessary for the maintenance of constant concentration in the system. It is seen that the function $V_{\Sigma}^{\mathrm{i}}=f(j)$ is linear in a wide range of changes in the technological

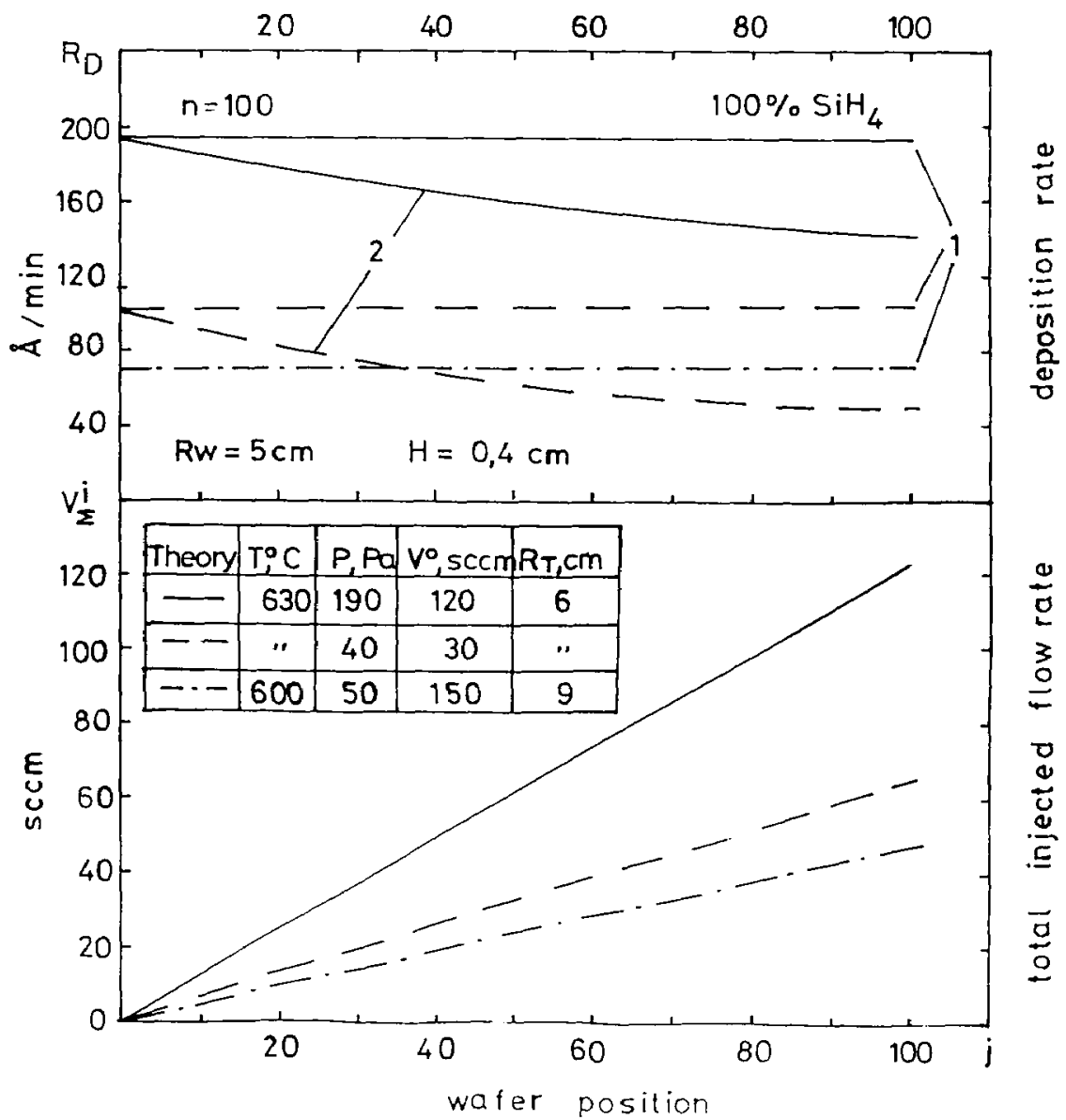

Fig. 7. Deposition rate $\left(R_{b}\right)$ of polysilicon and total injected flow rate of silane $\left(l_{\Sigma}^{\prime \prime}\right)$ as a function of wafer position $(j)$ for differcut walues of process parameters 
parameters.

Fig. 8 illustrates a similar comparison for an economically optimized regime [5], tracing the local gas flow $V_{\mathrm{g}}^{\mathrm{i}}(j)$ through the holes of the injector. It is seen that $V_{\mathrm{g}}^{\mathrm{i}}(j)$ does not change along the axis, and its value depends on the number of iniector holes $(\mathrm{m})$ along the reaction zone. The invariance of the function $V^{i}(j)$ is explained by the equality of the quantity of the injected active component and its consumption in accordance with the kinetic equation of the catalyzed process. That means that the results from the deposition should be independent of the presence or absence of main gas flow $\left(V^{\circ}\right)$ that is confirmed by the numerical experiments.

The conclusions drawn simplify considerably the design of the injectors when they are used in a regime of maintaining constant concentration in the reactor. The injectors can be likened to a tube with continuously distributed flow along the length through discreet holes (Fig.9). This case is fairly well investigated in practice [15].

\section{CONCLUSIONS}

The analysis of the suggested models shows a fair agreement between the predicted and practically used geometric characteristics of the deposition reactors. Since a vast experimental material is processed and analyzed during the design of such reactors, these results are an indirect measure of merit for their adequacy. The systems with injection feeding turn LPCVD reactors into ideal-mixing reactors. They operate in the most economical regime in view of the consumed reagents.

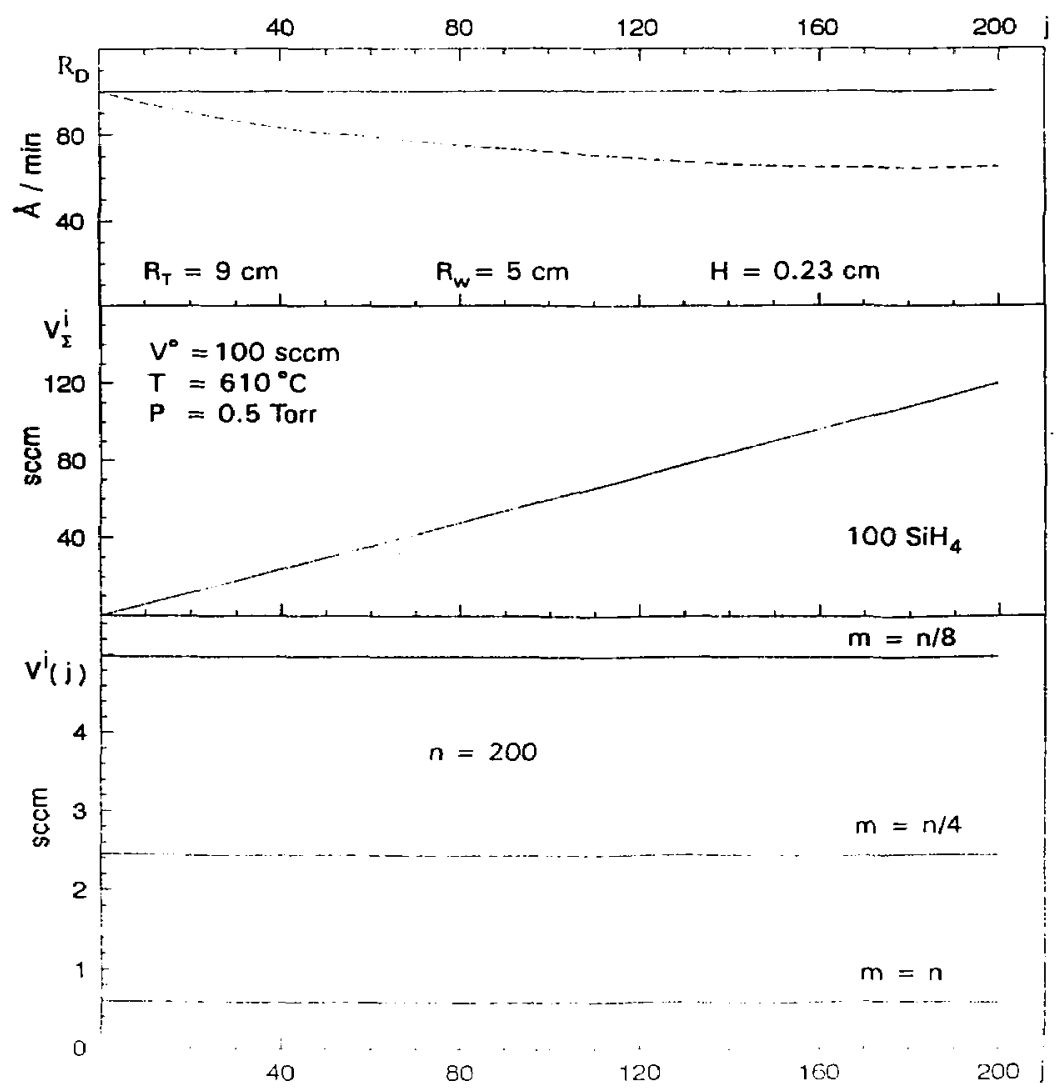

Fig. 8. Deposition rate of polysilicon $\left(R_{D}\right)$, total $\left(V_{Y}^{\prime}\right)$ and local $F^{\prime \prime}(\mathrm{j})$ flow rate of injected silane as a function of wafer position ( $j$ ) for an optimal choice of regime paramcters: with without injection. 


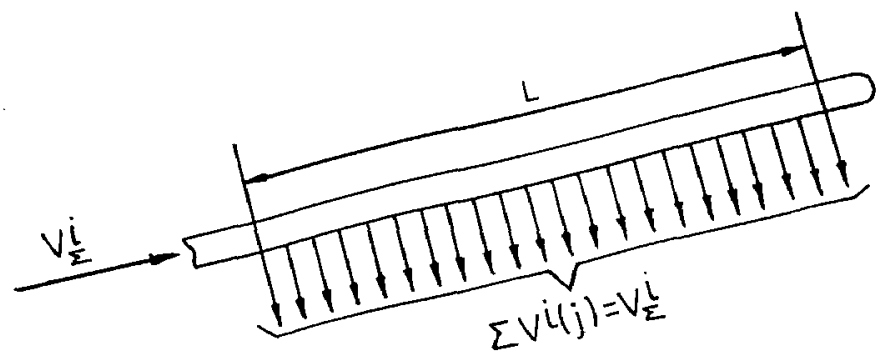

Fig. 9. Schematic of injector with continuously distributed flow along the length ( L ) through discreet holes.

\section{References}

[1] Condoret J.S., Constant G., Couderc J.P., Demengeot V, and Duverneuil P., Enthropie, No.125/126 (1985) 136.

[2] Jensen K. and Graves D., J. Electrochem. Soc., 130 (1983) 1950

[3] Yeckel A. and Middleman S. ibid., 134 (1987) 1275.

[4] Peev G., Zambov L, and Nedev 1., Thin Solid Films 190 (1990) 341.

[5] Peev G., Zambov L. and Yanakiev Y., J. Crystal Growth 106 (1990) 377.

[6] Peev G. and Zambov L., Thin Solid Films 203 (1991) 185

[7] Toor L. and Lee M., J. Crystal Growth 72 (1985) 679.

[8] Holleman J. and Middelhoek J., Thin Solid Films 114 (1984) 295.

[9] Juza J. and Cermak J., J. Electrochem. Soc. 129 (1982) 1627.

[10] Hess D., Jensen K. and Anderson T., Reviews in Chemical Engineering 3 (1985) 97.

[11] Zambov L., Vacuum 42 (1991) 1019.

[12] Bielle-Daspet D., Scheid E., Azzaro C., De Manduit B. and Pieraggi, B., Thin Solid Films 204 (1991) 33.

[13] Zambov L., Peev G., Shanov V. and Drumeva S., Vacuum 43 (1992) 227.

[14] Zambov L., J. Crystal Growth 125 (1992) 164.

[15] Magirski V., Hydrodynamics (Tehnika, Sofia, 1979) 335 (in Bulgarian).

\section{Appendix - List of Symbols}

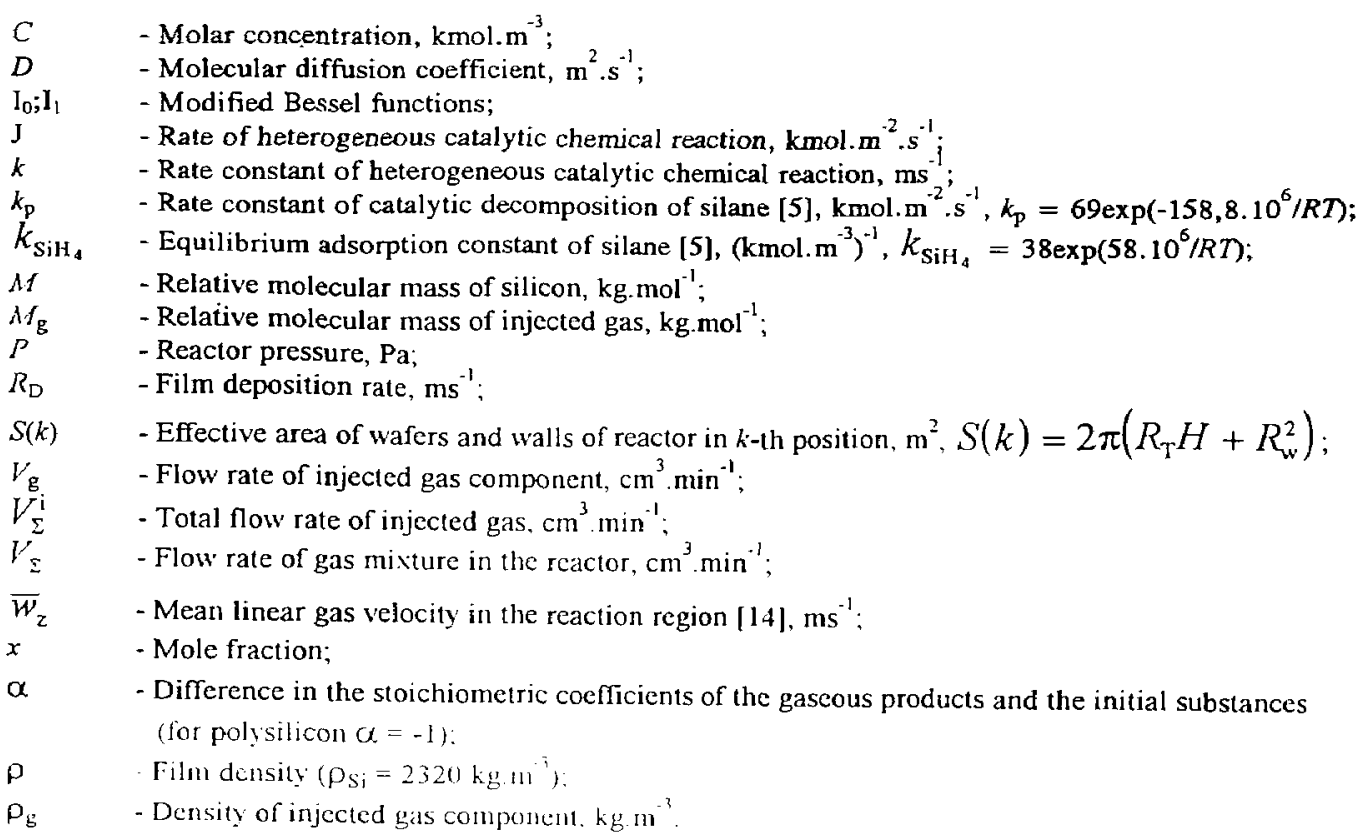

